Pour tenir compte de l'observation de Gứrault, le plus simple serait sans doute de définir trois sortes de Camemberts.

En supposant qu'on retienne comme base un extrait sec total de 110 grammes (1) environ, on aurait :

Le Camembert $40 \%$ de matière grasse dans l'extrait sec qui devrait avoir 45 grammes de matière grasse totale;

Le Camembert $45 \%$ de matière grasse dans l'extrait sec qui devrait avoir 50 grammes de matière grasse totale ;

Le Camembert $50 \%$ de matière grasse dans l'extrait sec qui devrait avoir 55 grammes de matière grasse totale.

En modifiant un peu l'article 2 du décret du 20 octobre 1936 , on pourrait appeler le premier : "Camembert", le second : "Camembert gras ", et le troisième : "Camembert extra-gras ». Nous avons personnellement toujours trouvé abusif d'appeler gras un fromage fabriqué avec un mélange de lait ne contenant guère que 25 grammes de matière grasse par litre.

Suivant la quantité d'extrait sec total que l'on veut leur voir contenir au début de l'affinage, on définirait de même trois variétés de "petits Camemberts".

\title{
DÉTERMINATION DES TENEURS EN EAU ET MATIERE SĖCHE, EN CALCIUM ET EN PHOSPHORE DE DIVERSES VARIÉTÉS DE FROMAGES FRAIS
}

\author{
par
}

\section{LUCIE RANDOIN et Colette JOURDAN-VATINEL}

\section{INTRODUCTION}

Après avoir déterminé les teneurs en calcium et en phosphore des principaux fromages à pâte molle [1] et à pâte ferme [2], nous avons étudié, à ce même point de vue, les fromages frais suivants :

(1) Ces fromages seront pratiquement plus petits que ceux que l'on fabriquait en 1939; nous pensons que c'est une erreur au point de vue économique car c'est pratiquement le nombre des fromages à pâte molle qui écrase souvent le marché et non leur poids. L'intérêt bien compris de la profession nous paraît donc être de diminuer ce nombre, d'autant plus qu'il faut proportionnellement moins de, main-d'œuvre, de boîtes d'emballage, etc..., quand les fromages sont plus gros.

Le camembert à $45 \%$ de matière grasse dans l'extrait sec fabriqué avant 1940 avait au moins 120 grammes d'extrait sec total. Peut-être pourrait-on se baser sur cette teneur ce qui donnerait :

Pour le $40 \%$ : 50 grammes de matière grasse totale.

Pour le $45 \%$ : 55 grammes de matière grasse totale.

Pour le $50 \%$ : 60 grammes de matière grasse totale. 
- fromage blanc du commerce,

- fromage blanc préparé au laboratoire sans présuré,

- fromage blanc préparé au laboratoire avec présure,

- fromage demi-sel,

- petit suisse.

Dans les publications scientifiques, il n'existe que très peu de chiffres concernant la valeur calcio-phosphorique des fromages frais. Nous avons déjà mentionné les résultats que G. GuittonNEAU et R. CheVAlier [3] avaient obtenu, en 1934, en déterminant les teneurs en calcium et en phosphore du fromage dit à la pie :

- en milligrammes dans l'extrait sec dégraissé en pour cent : calcium : $620 ;$ - phosphore : $990 ;$ - rapport $\mathrm{Ca} / \mathrm{P}: 0 / 62$;

ce qui, en milligrammes pour 100 grammes de matière fraîche, donne approximativement les chiffres suivants : calcium : 113; - phosphore : 180 ; - rapport Ca/P : 0,62.

\section{MÉTHODES DE DOSAGES}

Comme dans nos expériences précédentes, nous avons déterminé :

- les teneurs en eau et en matière sèche, par' séchage à l'étuve à $100^{\circ}$ jusqu'à poids constant;

- les teneurs en calcium par la méthode de Ch. 0. GuillauMIN [4];

- les teneurs en phosphore par la méthode de H. Copaux [5].

\section{RÉSULTATS EXPÉRIMENTAUX}

Tous nos résultats relatifs aux fromages frais figurent dans le tableau $I$, mais, ici encore, chaque teneur mentionnée est déjà une moyenne de deux chiffres qui se rapportent à deux portions identiques du même échantillon.

\section{Teneurs en eau et matière sèche.}

Dans les fromages étudiés, les teneurs en eau sont évidemment très fortes :

- les $2 / 3$ du poids frais total (environ $66 \%$ ) pour le demi-sel, le plus riche en matière sèche $(34 \%)$;

- les $3 / 4$ environ du poids frais pour le petit-suisse $(76 \%)$ et pour les deux sortes de fromages blancs préparés au laboratoire, l'un sans présure, l'autre avec présure (environ $74 \%$ );

- un peu plus des $4 / 5$ du poids frais pour le fromage blanc $d u$ commerce $(82 \%)$.

Rappelons : - que le pourcentage d'eau des fromages à pâte molle avec moisissures externes oscille entre $54 \%$ (Camembert) 
et $60 \%$ (Carré de l' Est); - que celui des fromages à pâte molle et croûte lavée est, en moyenne, de $46 \%$ (Livarot, Munster), et de $50 \%$ (Pont-l'Evêque); - enfin, que le pourcentage d'eau des fromages à pâte ferme est, en moyenne, de $34 \%$ pour le Gruyère, de 37 à $39 \%$ pour le Hollande et le Cantal, la teneur en eau du SaintPaulin $(48,6 \%$ en moyenne) s'écartant notablement de ces derniers chiffres.

\section{Teneurs en calcium.}

Etant donné les différences très notables qui existent entre les quantités d'eau contenues dans les diverses sortes de fromages frais, il nous semble préférable, pour que la comparaison entre leurs valeurs calcio-phosphoriques ait une signification bien nette, d'envisager surtout les teneurs en calcium et en phosphore qui se rapportent à la matière sèche, teneurs qui sont exprimées, dans le tableau $I$, en grammes de calcium (ou de phosphore) et, dans le tableau III (classement des fromages étudiés), en milligrammes de calcium (ou de phosphore).

Au point de vue de leur teneur en calcium, les einq sortes de produits frais que nous avons étudiées se partagent nettement comme suit :

$1^{\circ}$ le fromage blanc préparé au laboratoire avec présure qui est extrêmement riche en calcium, puisqu'il en renferme 1.489 milligrammes pour 100 grammes de produit sec;

$2^{\circ}$ un groupe relativement riche en calcium, qui comprend le fromage blanc du commerce et le fromage blanc préparé sans présure (respectivement 901 milligrammes et 831 milligrammes de calcium pour 100 grammes de produit sec);

$3^{\circ}$ enfin, un groupe moins riche en calcium, comprenant le petit-suisse et le demi-sel, lesquels ne renferment, le premier, que 429 milligrammes, et le second, que 303 milligrammes de calcium pour 100 grammes de matière sèche.

\section{Teneurs en phosphore.}

La même classification peut être faite après une comparaison portant sur les teneurs en phosphore (voir les tableaux I et III).

1. Le fromage blane préparé au laboratoire avec présure renferme de fortes quantités de phosphore: 765 milligrammes pour 100 grammes de matière sèche.

2. Le fromage blanc préparé au laboratoire sans présure et le fromage blanc du commerce en renferment des quantités moyennes, et même au-dessus de la moyenne : 575 milligrammes et 507 milligrammes.

3. Quant au petit-suisse et au demi-sel, ils sont moins riches en 
TABLEAU I

TENEUR EN GALGIUM ET EN PHOSPHORE DE DIVERSES VARIÉTÉS DE FROMAGES FRAIS

\begin{tabular}{|c|c|c|c|c|c|c|c|}
\hline \multirow{2}{*}{ Fromages } & \multirow{2}{*}{$\begin{array}{l}\text { Eau gr. } \\
\text { p. } 100\end{array}$} & \multirow{2}{*}{$\begin{array}{l}\text { Matière } \\
\text { sèche } \\
\text { (gr. p. } \\
100 \mathrm{gr} .)\end{array}$} & \multicolumn{2}{|c|}{$\begin{array}{c}\text { Caleium } \\
\text { (gr,p. } 100 \text { gr.) }\end{array}$} & \multicolumn{2}{|c|}{$\begin{array}{c}\text { Phosphore } \\
\text { (gr. p. } 100 \text { gr.) }\end{array}$} & \multirow{2}{*}{$\begin{array}{c}\text { Rapport } \\
\mathrm{Ca} / \mathrm{P}\end{array}$} \\
\hline & & & $\begin{array}{l}\text { de ma- } \\
\text { tière } \\
\text { fraîche }\end{array}$ & $\begin{array}{l}\text { de ma- } \\
\text { tière } \\
\text { sèche }\end{array}$ & $\begin{array}{l}\text { de ma- } \\
\text { tière } \\
\text { fraîche }\end{array}$ & $\begin{array}{l}\text { de ma- } \\
\text { tière } \\
\text { sèche }\end{array}$ & \\
\hline \multicolumn{8}{|l|}{$\begin{array}{c}\text { FROMAGE BLANC } \\
\text { du commerce: }\end{array}$} \\
\hline Ech. $1 \ldots \ldots \ldots$ & 82,53 & 17,47 & 0,104 & 0,594 & 0,071 & 0,405 & 1,47 \\
\hline Ech. $2 \ldots \ldots \ldots$ & 80,23 & 19,77 & 0,208 & 1,052 & 0,103 & 0,519 & 2,03 \\
\hline Ech. $3 \ldots \ldots \ldots$ & 83,45 & 16,55 & 0,175 & 1,057 & 0,099 & 0,596 & 1,77 \\
\hline Moyennes ...... & 82,07 & 17,93 & 0,162 & 0,901 & 0,091 & 0,507 & 1,77 \\
\hline \multicolumn{8}{|l|}{$\begin{array}{c}\text { Fromage BLANC } \\
\text { préparé au la- } \\
\text { boratoire sans } \\
\text { présure }(1) \text { : }\end{array}$} \\
\hline Ech. $1 \ldots \ldots \ldots$ & 71,10 & 28,90 & 0,260 & 0,899 & 0,125 & 0,432 & 2,08 \\
\hline Ech. $2 \ldots \ldots \ldots$ & 74,02 & 25,98 & 0,199 & 0,766 & 0,163 & 0,630 & 1,21 \\
\hline Ech. $3 \ldots \ldots \ldots$ & 75,60 & 24,40 & 0,219 & 0,897 & 0,169 & 0,690 & 1,29 \\
\hline Ech. $4 \ldots \ldots \ldots$ & 73,44 & 26,56 & 0,202 & 0,760 & 0,146 & 0,549 & 1,38 \\
\hline Moyennes..... & 73,54 & 26,46 & 0,220 & 0,831 & 0,151 & 0,575 & 1,45 \\
\hline \multicolumn{8}{|l|}{$\begin{array}{c}\text { Fromage BLANC } \\
\text { préparé au la- } \\
\text { boratoire avec } \\
\text { présure }(1):\end{array}$} \\
\hline Ech. $1 \ldots \ldots \ldots$ & 71,24 & 28,76 & 0,398 & 1,380 & 0,171 & 0,593 & 2,32 \\
\hline Ech. $2 \ldots \ldots \ldots$ & 76,81 & 23,19 & 0,400 & 1,725 & 0,218 & 0,940 & 1,83 \\
\hline Ech. $3 \ldots \ldots \ldots$ & 74,41 & 25,59 & 0,394 & 1,539 & 0,209 & 0,816 & 1,88 \\
\hline Ech. $4 \ldots \ldots \ldots$ & 72,34 & 27,66 & 0,363 & 1,312 & 0,197 & 0,712 & 1,84 \\
\hline Moyennes...... & 73,70 & 26,30 & 0,389 & 1,489 & 0,199 & 0,765 & 1,95 \\
\hline \multicolumn{8}{|l|}{$\begin{array}{l}\text { Petit-suisse du } \\
\text { eommerce: }\end{array}$} \\
\hline Eeh. $1 \ldots \ldots \ldots$ & 73,60 & 26,40 & 0,123 & 0,466 & 0,104 & 0,394 & 1,18 \\
\hline Ech. $2 \ldots \ldots \ldots$ & 75,04 & 24,96 & 0,112 & 0,449 & 0,074 & 0,296 & 1,51 \\
\hline Ech. $3 \ldots \ldots \ldots$ & 75,13 & 24,87 & 0,102 & 0,410 & 0,090 & 0,361 & 1,13 \\
\hline Ech. $4 \ldots \ldots \ldots$ & 75,90 & 24,10 & 0,108 & 0,448 & 0,095 & 0,394 & 1,13 \\
\hline Ech. $5 \ldots \ldots \ldots$ & 80,00 & 20,00 & 0,074 & 0,370 & 0,079 & 0,395 & 0,92 \\
\hline Moyennes ..... & 75,93 & 24,07 & 0,104 & 0,429 & 0,088 & 0,368 & 1,17 \\
\hline \multicolumn{8}{|l|}{$\begin{array}{l}\text { DEMI-SEL du com- } \\
\text { merce : }\end{array}$} \\
\hline Ech. $1 \ldots \ldots \ldots$ & 69,70 & 30,30 & 0,105 & 0,347 & 0,068 & 0,224 & 1,50 \\
\hline Ech. $2 \ldots \ldots \ldots$ & 66,76 & 33,24 & 0,089 & 0,267 & 0,073 & 0,228 & 1,17 \\
\hline Ech. $3 \ldots \ldots \ldots$ & 64,64 & 35,36 & 0,079 & 0,224 & 0,075 & 0,215 & 1,04 \\
\hline Ech. $4 \ldots \ldots \ldots$ & 64,40 & 35,60 & 0,125 & 0,351 & 0,106 & 0,297 & 1,18 \\
\hline Ech. $5 \ldots \ldots \ldots$ & 63,89 & 36,11 & 0,118 & 0,326 & 0,095 & 0,263 & 1,24 \\
\hline Moyennes..... & 65,88 & 34,12 & 0,103 & 0,303 & 0,083 & 0,245 & 1,23 \\
\hline
\end{tabular}

(1) Les échantillons de fromages blancs préparés avec ou sans présure qui portent le même numéro ont été obtenus à partir du même lait. 
phosphore que les précédents, car ils renferment seulement : 368 milligrammes et 245 milligrammes pour 100 grammes de matière sèche.

\section{Valeurs du rapport $\mathrm{Ca} / \mathbf{P}$.}

L'examen comparatif, - sur les tableaux I, II et III - des valeurs du rapport $\mathrm{Ca} / \mathrm{P}$, conduit encore à placer au premier rang le fromage blanc préparé avec présure (valeur du rapport $\mathrm{Ca} / \mathrm{P}: 1,95$ ).

Viennent ensuite le fromage blanc du commerce $(1,77)$, puis le fromage blanc préparé sans présure $(1,45)$.

Enfin les valeurs du rapport $\mathrm{Ca} / \mathrm{P}$, pour le demi-sel et le petitsuisse, sont respectivement de 1,23 et 1,17 . Certes, elles se rapprochent de celles qui caractérisent le lait de vache, mais on voit qu'elles sont, malgré tout, inférieures à la plupart des valeurs du rapport $\mathrm{Ca} / \mathrm{P}$ que nous avons mentionnées jusqu'ici. Il n'y aurait d'exceptions qu'en ce qui concerne certains fromages à pâte molle : Brie, Camembert et Carré de l'Est $(0,97,1,09$ et 1,15$)$.

\section{CONCLUSIONS}

En alimentation rationnelle, les fromages frais représentent une source précieuse de calcium et, dans une moindre mesure, de phosphore. De ce fait, ils ont l'avantage de pouvoir contribuer très efficacement à compléter et à rééquilibrer une ration renfermant pain, pâtes, pommes de terre, viande ou œufs, - aliments qui sont, au contraire, pauvres en calcium et riches en phosphore -, de telle sorte que la valeur du rapport $\mathrm{Ca} / \mathrm{P}$ de la ration quotidienne puisse, par mesure de précaution, atteindre 1 environ pour les enfants, les adolescents, les femmes enceintes et allaitantes, et 0,7 pour les adultes.

Les fromages blancs préparés, dans le laboratoire, avec de la présure sont particulièrement riches en calcium et en phosphore (respectivement I gr., 50 et 0 gr., 76 pour 100 grammes secs), et leur rapport $\mathrm{Ca} / \mathrm{P}$ est très élevé (environ 2). Par rapport au poids sec, ils renferment presque autant de calcium que le Gruyère et notablement plus que le Livarot, le Cantal, le Hollande, le Saint-Pautin, le Pont-l'Evéque, tous fromages qui comptent pourtant, - nous l'avons mentionné dans nos précédents mémoires -, parmi les aliments les plus riches en calcium.

Les fromages blancs préparés, dans le laboratoire, sans présure, à partir des mêmes laits que les précédents, sont toujours moins riches en calcium et en phosphore (respectivement 0 gr., 83 et 0 gr., 57 pour 100 grammes secs), et leur rapport $\mathrm{Ca} / \mathrm{P}$ est moins élevé (en moyenne 1,45). Ce phénomène doit évidemment être mis en relation avec la lenteur de la coagulation en l'absence de présure, 
TABLEAU II

GLASSEMENT DES FROMAGES FRAIS ÉTUDIÉS (MATIÈRE FRAICHE)

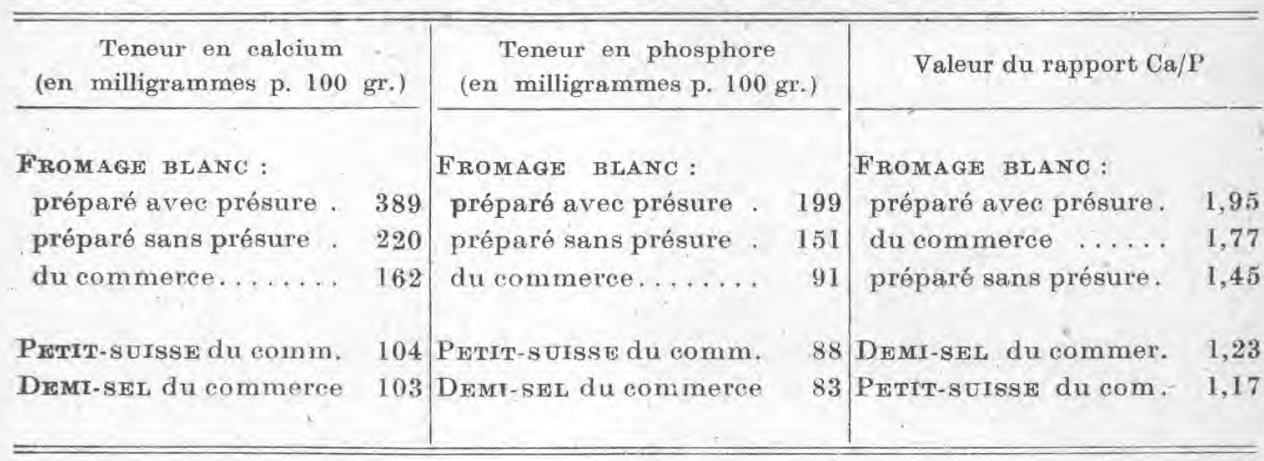

TABLEAU III

CLASSEMENT DES FROMAGES ÉTUDIÉS (MATIERE SÈCHE)

\begin{tabular}{|c|c|c|c|c|c|}
\hline $\begin{array}{c}\text { Teneur en calcium } \\
\text { (en milligrammes p. } 100\end{array}$ & gr.) & $\begin{array}{l}\text { Teneur en phosphore } \\
\text { (en milligrammes p. } 100\end{array}$ & & Valeur du rapport $\mathrm{Ca} /$ & \\
\hline \multirow{2}{*}{\multicolumn{2}{|c|}{$\begin{array}{l}\text { Fromage BLANC : } \\
\text { préparé avee présure . } 1.489\end{array}$}} & \multirow{2}{*}{\multicolumn{2}{|c|}{$\begin{array}{l}\text { Fromage BLANC : } \\
\text { préparé avec présure : } 765\end{array}$}} & \multicolumn{2}{|l|}{ FROMAGE BLANC : } \\
\hline & & & & préparé avec présure. & 1,95 \\
\hline du commerce....... & 901 & préparé sans présure. & 575 & du commeree ...... & 1,77 \\
\hline préparé sans présure. & 831 & du commerce........ & 507 & préparé sans présure. & 1,45 \\
\hline PeTIT-SUISSE du comm. & 429 & PETIT-SUISSE du comm. & 368 & DEMI-SELducommerce. & 1,23 \\
\hline DEMI-SEL du commerce. & 303 & DEMI-SEL du commerce. & 245 & Petit-suisse du comm. & 1,17 \\
\hline
\end{tabular}

lenteur qui permet une acidification plus accusée du caillé, et entraîne, de ce fait, le passage d'une quantité accrue de phosphore, et surtout de calcium, dans le lactosérum.

L'intérêt des fromages blancs du commerce comme sources de calcium et de phosphore varie d'un échantillon à un autre. Dans l'ensemble cependant, les échantillons que nous avons analysés se sont avérés inférieurs à ceux que nous avons préparés au laboratoire avec de la présure, ceci, aussi bien du point de vue de leurs teneurs absolues en calcium et en phosphore, qu'en ce qui concerne leur rapport ealcio-phosphorique.

Quant aux petits-suisses et aux demi-sels, ils sont moins riches en calcium et en phosphore que les fromages blancs et leur rapport calcio-phosphorique est moins élevé. Pour 100 grammes secs, les petits-suisses du commerce renferment en moyenne, 0 gr., 43 de calcium et 0 gr., 37 de phosphore: leur rapport $\mathrm{Ca} / \mathrm{P}$ est de près de 1,2 . Pour 100 grammes secs également, les demi-sels du commerce contiennent, en moyenne, 0 gr., 30 de calcium et 0 gr., 24 de phosphore; 
leur rapport $\mathrm{Ca} / \mathrm{P}$ est du même ordre de grandeur que celui des petits-suisses.

On voit que certains fromages frais peuvent contribuer, pour une large part, à la couverture des besoins calciques de notre organisme. Ainsi, pour remplacer, à ce point de vue, $1 / 4$ de litre de lait, il suffirait de consommer 80 grammes d'un fromage blanc très riche en calcium, tel que celui que nous avons préparé nous-mêmes au laboratoire avec de la présure.

(Travail du Laboratoire de Physiologie de la Nutrition du Centre National de la Recherche Scientifique, et de l'Institut National de la Recherche agronomique).

\section{BIBLIOGRAPHIE}

[1] L. Randorn et C. VAtinel. Le Lait, 1951, 31, 113.

[2] L. Randoin et C. Jourdan-Vatinel. Le Lait, 1951, 31, 250.

[3] G. Guittonneau et R. Chevalier. C. R. Ac. Sc., 1934, 199, 801.

[4] Ch. O. Guillaumin. Bull. Soc. Ohim. biol., 1932, 14, 105.

[5] H. Copaux. C. R. Ac. Sc., 1921, 173, 656.

[6] L. Randoin, P. Le Gallic et J. Causeret. Tables de composition des aliments ( $2^{\mathrm{e}}$ édition), précédées des Normes de l'alimentation humaine. Lanore, éditeur. Paris, 1947.

\section{SUR LA FORMATION DE L'AROME DU BEURRE} par

\section{A. C. F. KRUIJER}

Bien que le dernier mot n'ait pas été dit en ce domaine et qu'il $\mathrm{y}$ ait des points de vue complémentaires à attendre, il est peut-être justifiable de résumer la théorie de Van Beynum ét Pette, extraite de leur livre "Microbiologie laitière et hygiène du lait ", écrit en collaboration avec SJollema (1).

Cette théorie se résume ainsi : le lait contient jusqu'à 2 grammes d'acide citrique $\left(\mathrm{C}^{6} \mathrm{H}^{8} \mathrm{O}^{7}\right.$. $\left.\mathrm{H}^{2} \mathrm{O}\right)$ par litre, décomposé par l'action conjuguée du Betacoccus cremoris et des streptocoques Lactis et Cremoris; les facteurs qui dirigent cette décomposition sont le degré d'acidité du milieu et la présence ou l'absence d'oxygène. Indépendamment de l'acidité et de l'oxygène, les deux premières étapes s'exécutent ainsi :

(1) J. Van Beynum, J. W. Pette et P. Sjollema. Zuivelmicrobiologie en Melkhygiene, Editeur : C. Misset N. V. - Doetinchen, Hollande, 1950. 